\title{
La violencia, el estrés y el asma
}

\author{
Violence, stress, and asthma
}

Juan C. Celedón, MD., PhD.

El asma es un grave problema de salud pública en Colombia (1) y en el mundo (2). En un estudio reciente de 5.978 sujetos en seis ciudades colombianas, aproximadamente el $17 \%$ de los niños (entre 5 y 17 años) y el $9 \%$ de los adultos (entre 18 y 59 años) participantes, reportaron haber tenido sibilancias en el año previo a la encuesta (1). En ese estudio, 321 $(\sim 43 \%)$ de 747 participantes (con edades entre 1 y 59 años) que reportaron sibilancias, habían acudido a un departamento de urgencias o requerido al menos una hospitalización por asma en el año previo (1).

Alrededor del mundo, la prevalencia del asma y la morbilidad causada por esta varían de acuerdo con una serie de características, incluyendo edad, sexo, etnicidad, lugar de residencia, y estrato socioeconómico (3, 4). Aunque las causas de esta "epidemia global de asma" o de las inequidades en la prevalencia del asma son multi-factoriales e insuficientemente definidas, podrían incluir cambios en el número de nacimientos por cesárea, dieta, calidad del aire ambiental, exposición a microbios o virus en la vida temprana, tabaquismo de segunda mano, sobrepeso u obesidad y estrés psicosocial (a causa del estilo de vida contemporáneo y la exposición a eventos traumáticos).

El trastorno por estrés post-traumático (TEPT) y el estrés, se han asociado con el asma o la morbilidad causada por esta en niños y adultos $(5,6)$. En Estados Unidos, los puertorriqueños y los afro-americanos están tan desproporcionadamente afectados por el asma, como expuestos a la violencia y a eventos traumáticos (5). De igual forma, los puertorriqueños también podrían ser más susceptibles a eventos traumáticos $(7,8)$. Cuando se les compara con miembros de otros grupos étnicos, los puertorriqueños tuvieron un riesgo mayor de desarrollar síntomas del TEPT luego de los ataques terroristas del 11 de septiembre de 20017.

Hallazgos recientes sugieren que los eventos violentos o traumáticos contribuyen a la alta morbilidad por asma en puertorriqueños u afro-americanos (9-11). Aunque la exposición al estrés materno durante la infancia se ha asociado continuamente con morbilidad por asma en niños de origen distinto al puertorriqueño (12,
División de Neumología, Alergia e Inmunología, Children's Hospital of Pittsburgh of the University of Pittsburgh Medical Center, University of Pittsburgh, Pittsburgh, PA.

Correspondencia: Juan C. Celedón. Division of Pulmonary Medicine, Allergy and Immunology. Children's Hospital of Pittsburgh of UPMC. 4401 Penn Avenue, Pittsburgh, PA 15224 Teléfono: 412.692.8429; Fax: 412.692.7636. Correo electrónico: juan.celedon@chp.edu Recibido: 27-08-2013. Aceptado: 13-11-2013. 
13), se sabe relativamente poco sobre el estrés paterno y el asma infantil. En el primer estudio sobre el estrés tanto paterno como materno, realizado en mellizos puertorriqueños a quienes se les hizo seguimiento entre las edades de 1 y 3 años, nuestro grupo demostró que la depresión paterna está asociada con el uso de corticosteroides orales para el asma, así como que la depresión en el padre o en la madre se relaciona con mayor riesgo de hospitalizaciones por asma (14). En un estudio de corte transversal en niños puertorriqueños de edad escolar, nuestro grupo también fue el primero en reportar que el abuso físico o sexual está asociado con un riesgo mayor de asma o morbilidad por asma (9). Este hallazgo fue confirmado recientemente y extendido por otros investigadores en un estudio longitudinal de mujeres afro-americanas, en quienes el abuso físico durante la niñez estuvo asociado con mayor riesgo de desarrollar asma durante la vida adulta (11). Hasta la fecha, los mecanismos que pudiesen explicar la asociación entre el estrés y el asma son en su mayoría desconocidos (5).

El medio ambiente y el estilo de vida pudiesen causar asma no solo a través de interacciones con variantes genéticas sino también de mecanismos epigenéticos como la metilación de ADN (15). Dicho proceso implica una modificación reversible del ADN, a través de la adición de un grupo metilo a la posición 5 ' de un residuo de citosina, lo cual a menudo causa una reducción en los niveles de expresión de un gen (16). A diferencia de la secuencia de ADN, los niveles de metilación del ADN varían entre los diferentes compartimientos celulares en los tejidos humanos, como en el pulmón o el hígado.

Recientemente, un estudio seminal que utilizó ADN de leucocitos demostró que la metilación de un gen que regula las respuestas al estrés (el receptor 1 de la ciclasa de adenilato pituitario [ADCYAPIRI]) se asocia con el TEPT en adultos (17). Este y un estudio adicional, también demostraron que un polimorfismo de nucleótido simple (SNP) en $A D C Y A$ P1Rl (rs2267735) se asocia con el TEPT en mujeres adultas y con la ansiedad en niños $(17,18)$. Aunque un estudio de adultos estadounidenses (expuestos a condiciones menos estresógenas que aquellos en el estudio original) no encontró una asociación entre el
SNP rs2267735 y el TEPT (19), no se intentó replicar los hallazgos referentes a la metilación de $A D$ CYAPIR1.

Con base en nuestros hallazgos previos, se realizó un estudio reciente sobre la exposición a la violencia, variantes genéticas y epigenéticas en $A D$ CYAPIRl, y asma en niños puertorriqueños (20). Como parte de este trabajo, se encontró una asociación significativa entre la metilación de un sitio $\mathrm{CpG}$ (cg11218385) en el promotor de ADCYAPIRl y el asma infantil en puertorriqueños. En niños puertorriqueños, la exposición a la violencia se asoció no solo con el asma (índice de probabilidad ajustado [aOR] por cada incremento de 1 punto en la escala de exposición a la violencia $=1,1$, intervalo de confianza del $95 \%[\mathrm{CI}]=1,0-1,2, \mathrm{p}<0,05)$ sino también con mayor metilación del cg11218385. Más aún, el alelo C del SNP rs2267735 estuvo asociado con el asma, independientemente de la metilación del $\operatorname{cg} 11218385(\mathrm{aOR}=1,3,95 \% \mathrm{CI}=1,0-1,7, \mathrm{p}<0,05)$. Este es el primer estudio que implica la variación genética y epigenética en un gen que confiere susceptibilidad al TEPT o la ansiedad (ADCYAPIRI) en la causalidad del asma (21).

Aunque nuestros hallazgos sobre ADCYAPIRI (20) deben ser interpretados cuidadosamente mientras se realizan estudios de replicación y estudios de corte longitudinal, estos sirven como apoyo preliminar a la hipótesis de que la exposición a la violencia influencia el inicio del asma, sea de forma directa (e.g. en individuos que son susceptibles dadas sus variantes genéticas) o indirecta (e.g. a través de la metilación del ADN). Es muy probable, sin embargo, que el estrés o la exposición a la violencia también influencien el desarrollo del asma por medio de otros mecanismos, incluyendo anormalidades en inmuno-regulación, percepción alterada de síntomas y falta de adherencia o uso regular de las medicaciones prescritas para el asma (5).

A pesar de avances los recientes, aún queda mucho por hacer para aumentar nuestra comprensión sobre el papel (si hay alguno) del estrés psicosocial en la patogénesis del asma o la morbilidad por asma. Como parte de estos esfuerzos, se lleva a cabo un estudio sobre el estrés, la epigenética y el asma en una 
gran cohorte de niños puertorriqueños. Pese a la realización de estudios como este y otros, en minorías étnicas y poblaciones marginadas en países desarrollados como los Estados Unidos, hay una necesidad tanto real como urgente de entender la relación entre la exposición a la violencia, el estrés y el asma en países latino-americanos como Colombia, donde estas condiciones son relativamente comunes.

El objetivo final de los nuevos estudios investigativos es identificar nuevos biomarcadores (e.g. epigenéticos) del asma en individuos que han sido expuestos a eventos violentos o altamente traumáticos. Este hecho debe, a su vez, llevar al desarrollo de nuevos medios para la prevención (e.g. a través de la reducción del estrés paterno o materno), diagnóstico (e.g., al permitir la diferenciación [particularmente en niños pequeños] entre síntomas que pareciesen deberse al asma y aquellos realmente causado por esta) y al tratamiento (e.g., si se pudiese revertir la metilación a través de medios farmacológicos o nofarmacológicos) del asma relacionada con el estrés.

\section{Bibliografía}

1. Dennis RJ, Caraballo L, Garcia E, et al. Prevalence of asthma and other allergic conditions in Colombia 2009-2010: a cross-sectional study. BMC Pulm Med. 2012;12:17.

2. Asher MI, Montefort S, Bjorksten B, et al. Worldwide time trends in the prevalence of symptoms of asthma, allergic rhinoconjunctivitis, and eczema in childhood: ISAAC Phases One and Three repeat multicountry cross-sectional surveys. Lancet. 2006;368:733-43.

3. Akinbami LJ, Moorman JE, Liu X. Asthma prevalence, health care use, and mortality: United States, 2005-2009. Natl Health Stat Report. 2011:1-14.

4. Forno E, Celedon JC. Health disparities in asthma. Am J Respir Crit Care Med. 2012;185:1033-5.

5. Yonas MA, Lange NE, Celedon JC. Psychosocial stress and asthma morbidity. Curr Opin Allergy Clin Immunol. 2012;12:202-10.

6. Spitzer C, Koch B, Grabe HJ, et al. Association of airflow limitation with trauma exposure and post-traumatic stress disorder. Eur Respir J. 2011;37:1068-75.
7. Galea S, Vlahov D, Tracy M, Hoover DR, Resnick H, Kilpatrick D. Hispanic ethnicity and post-traumatic stress disorder after a disaster: evidence from a general population survey after September 11, 2001. Ann Epidemiol. 2004;14:520-31.

8. Ortega AN, Rosenheck R. Posttraumatic stress disorder among Hispanic Vietnam veterans. Am J Psychiatry. 2000;157:615-9.

9. Cohen RT, Canino GJ, Bird HR, Celedon JC. Violence, abuse, and asthma in Puerto Rican children. Am J Respir Crit Care Med. 2008;178:453-9.

10. Lange NE, Bunyavanich S, Silberg JL, Canino G, Rosner BA, Celedon JC. Parental psychosocial stress and asthma morbidity in Puerto Rican twins. J Allergy Clin Immunol. 2011;127: 734-40.

11. Coogan PF, Wise LA, O'Connor GT, Brown TA, Palmer JR, Rosenberg L. Abuse during childhood and adolescence and risk of adultonset asthma in African American women. J Allergy Clin Immunol. 2013;131: 1058-63.

12. Wood RA, Bloomberg GR, Kattan M, et al. Relationships among environmental exposures, cord blood cytokine responses, allergy, and wheeze at 1 year of age in an inner-city birth cohort (Urban Environment and Childhood Asthma study). J Allergy Clin Immunol 2011;127:913-9 e1-6.

13. Sharp LK, Curtis LM, Mosnaim G, Shalowitz MU, Catrambone C, Sadowski LS. The influence of caregiver's psychosocial status on childhood asthma and obesity. Annals of Allergy, Asthma, \& Immunology 2009;103:386.

14. Lange NE, Bunyavanich S, Silberg JL, Canino G, Rosner BA, Celedón JC. Parental psychosocial stress and asthma morbidity in Puerto Rican twins. Journal of Allergy and Clinical Immunology. 2011;127:734-40.e7.

15. Ho SM. Environmental epigenetics of asthma: an update. J Allergy Clin Immunol. 2010;126:453-65.

16. Durham AL, Wiegman C, Adcock IM. Epigenetics of asthma. Biochim Biophys Acta. 2011;1810: 1103-9.

17. Ressler KJ, Mercer KB, Bradley B, et al. Post-traumatic stress disorder is associated with PACAP and the PAC1 receptor. Nature. 2011;470:492-7.

18. Jovanovic T, Norrholm SD, Davis J, et al. PAC1 receptor (ADCYAP1R1) genotype is associated with dark-enhanced startle in children. Mol Psychiatry. 2013;18:742-3.

19. Chang SC, Xie P, Anton RF, et al. No association between ADCYAP1R1 and post-traumatic stress disorder in two independent samples. Mol Psychiatry 2012;17:239-41.

20. Chen W, Boutaoui N, Brehm JM, et al. ADCYAP1R1 and Asthma in Puerto Rican Children. Am J Respir Crit Care Med. 2013;187: 584-8.

21. Murphy SK, Hollingsworth JW. Stress: a possible link between genetics, epigenetics, and childhood asthma. Am J Respir Crit Care Med 2013;187:563-4. 